Pacific Journal of Mathematics

DERIVATION IN INFINITE PLANES 


\section{DERIVATION IN INFINITE PLANES}

\section{N. L. JOHNSON}

The purpose of this article is to study "derivation" in arbitrary affine planes. It is shown that the derivation process extends to arbitrary planes which possess a suitable set of Baer subplanes.

1. Introduction. A basic problem of interest is developing Ostrom's finite net replacement theory in the infinite case. Some expected premiums could be that the procedures are valid in infinite planes which have no finite analogue. For example, the Moufang planes, non-Pappian Desarguesian planes, and certain Bol planes may permit net replacement (see $\S 4$ ).

The present article will be restricted to studying derivation in infinite planes. Concerning infinite planes, Rosati [18] found a class of infinite Hughes planes and Swift [21] remarked that derivation is probably valid in infinite Pappian planes. This statement was essentially confirmed by Pickert [17] who also gave an algebraic construction of the Ostrom-Rosati planes (see Panella [15]).

Sabharwal [20] constructed a class of infinite André nearfield planes and showed that derivation is valid in these planes and also considered the analogous infinite "derivable chains" of Fryxell [6].

Barlotti and Bose [3] have studied the derivation of dual translation planes of dimension 2 by means of linear representations in projective spaces of projective planes (see [3], [4], [5]). The Bose-Barlotti derivation theory is valid in all dual translation planes of dimension 2 whose associated spread of the corresponding translation plane is also a dual spread. However, this condition is not valid in every infinite dual translation plane of dimension 2 (see [7]).

This article will be devoted to derivation in arbitrary planes. The treatment is in the spirit of Ostrom's original construction (see [13], section III, and [14]). Section 2 is devoted to showing that the derivation process extends to arbitrary planes which possess a suitable set of Baer (see (2.1)) subplanes. Section 3 is concerned with certain conditions sufficient for a given subplane to be a Baer subplane and develops some theory related to the derivation of translation planes and their duals. Finally, applications of the theory to certain infinite planes are considered in $\S 4$.

The author would like to express his appreciation to the referee for many helpful suggestions as to the form of this paper.

2. The Construction. Ostrom ([13] section III, pp. 7, 8,9) 
develops derivation in finite planes. The planes are of order $q^{2}$ and the procedure involves the relabeling of certain subplanes of order $q$ (Baer subplanes) as lines. Ostrom's arguments depend strongly on finiteness. However, it will be shown that the essential assumption is not of finiteness but is simply that the subplanes used in the process are Baer subplanes.

Definition 2.1. Let $\pi$ be a projective plane. A proper subplane $\pi_{0}$ of $\pi$ is a Baer subplane of $\pi$ if and only if every point of $\pi$ is on a line of $\pi_{0}$ and every line of $\pi$ is on a point of $\pi_{0}$.

\section{REMARK 2.2. A Baer subplane is maximal.}

Proof. Let $\pi_{0}$ be a Baer subplane of a projective plane $\pi$ and let $P^{*}$ be a point of $\pi-\pi_{0}$. Any subplane $\tau$ of $\pi$ containing $P^{*}$ and $\pi_{0}$ contains the joins of $P^{*}$ with points of $\pi_{0}$. Let $l$ be an arbitrary line of $\pi$ incident with $P^{*}$. By assumption $l$ intersects $\pi_{0}$ and therefore $\tau$ contains all lines of $\pi$ incident with the point $P^{*}$. Similarly, $\tau$ contains all lines of $\pi$ incident with any point of $\tau-\pi_{0}$. Let $Q$ be a point of $\pi$. Every line of $\pi$ incident with $Q$ intersects $\tau$. If $Q \notin \tau$ then $Q P^{*}$ is either a line of $\tau-\pi_{0}$ or is the unique line of $\pi_{0}$ incident with $P^{*}$. Since there is a line of $\pi_{0}$ incident with $Q$, it follows that $Q \in \tau$ in the former case. In the latter case, if $R$ is a point of $Q P^{*}$, choose a quadrangle whose cross joins contain $R$. Thus, all points of $\pi$ are in $\tau$.

DEFINITION 2.3. Let $\pi$ be a projective plane. Let $l_{\infty}$ be a line of $\pi$. A derivation set $\delta$ in $l_{\infty}$ is a set of points of $l_{\infty}$ such that if $P, Q$ are distinct points of $\pi-l_{\infty}$ such that $P Q \cap l_{\infty} \in \delta$ then there is a Baer subplane $\pi_{P, Q, \delta}$ of $\pi$ containing $P, Q, \delta$ such that $\delta$ is a line of $\pi_{P, Q, \hat{o}} \cdot$

We shall assume in the following that $\delta$ is a derivation set in $l_{\infty}$ for a projective plane $\pi$ and $\pi_{P, Q, \delta}$ is a Baer subplane containing $P, Q$ and $\delta$ as a line where $P Q \cap l_{\infty} \in \delta$. Also a point of $\pi_{P, Q, \delta}-\delta$ will be called an affine point of $\pi_{P, Q, \tilde{o}}$.

LEMMA 2.4. $\pi_{P, Q, o}$ is the unique proper subplane containing $P$ and $Q$ which contains $\delta$ as a line.

Proof. Let $\Sigma_{P, Q, \delta}$ be any subplane of $\pi$ containing points $P, Q$ which contains $\delta$ as a line. Let $P Q \cap l_{\infty}=\delta_{1} \in \delta$. Let $T=\left\{Q \delta_{2} \cap P \delta_{3}\right.$ where $\delta_{1}, \delta_{2}, \delta_{3}$ are distinct elements of $\left.\delta\right\}$. Let $\pi_{0}=\left\{Q \delta_{i} \cap P \delta_{j} \mid \forall \delta_{i}\right.$, $\delta_{j} \in \delta$ and not both $\delta_{i}, \delta_{j}$ equal to $\left.\delta_{1}\right\} \cup\left\{Q \delta_{i} \cap T \delta_{j} \mid \forall \delta_{i}, \delta_{j} \in \delta\right.$ and not 
both $\delta_{i}, \delta_{j}$ equal to $\left.\delta_{2}\right\}$.

Assume there is a point $R \in \Sigma_{P, Q, \delta}-\pi_{0}$. Since $P, Q, R \in \Sigma_{P, Q, \delta}$, either $R P \cap l_{\infty}$ and $R Q \cap l_{\infty}$ are distinct points in $\delta$ or $P, Q, R$ are collinear. In the latter case, $R=P\left(R P \cap l_{\infty}\right) \cap T\left(R T \cap l_{\infty}\right)$ and in the former $R=P\left(R P \cap l_{\infty}\right) \cap Q\left(R Q \cap l_{\infty}\right)$. Therefore, the point sets of $\Sigma_{P, Q, \tilde{o}}$ and $\pi_{0}$ are equal since clearly $\pi_{0} \leqq \Sigma_{P, Q, \tilde{o}}$.

Similarly, let $\tilde{\pi}_{0}=\left\{M \delta_{i} \mid M \in \pi_{0} \forall \delta_{i} \in \delta\right\}$. If $l$ is a line of $\Sigma_{P, Q, \delta}$ then $l \cap l_{\infty} \in \delta$ and there are at least two distinct points $L, U \in\left(l-l \cap l_{\infty}\right) \cap$ $\pi_{P, Q, \delta}$. Thus, $L, U \in \pi_{0}-\delta$ so that $l \in \tilde{\pi}_{0}$.

$\therefore$ Any two subplanes of $\pi$ which contain points $P$ and $Q$ and contain $\delta$ as a line have the same point sets and the same line sets and hence are identical.

Lemma 2.5. Any two points of $\pi_{P, Q, \tilde{o}}-\delta$ uniquely determine the subplane. Thus, any two distinct Baer subplanes $\pi_{P, Q, \delta}$ and $\pi_{R, S, \delta}$ intersect in $\delta$ or $\delta \cup\{M\}$ for some affine point $M$.

Proof. Let $M, S$ be any two distinct points of $\pi_{P, Q, \dot{\delta}}-\delta$. If $M$ and $S$ are $\notin P Q, \exists \delta_{i}, i=1,2,3,4, \in \delta \ni M=P \delta_{1} \cap Q \delta_{2}, S=P \delta_{3} \cap Q_{4}$. Clearly $P, Q \in \pi_{M, S, \delta}$ so that $\pi_{P, Q, \delta}=\pi_{M, S, \delta}$ by (2.4).

The remaining situations where $M$ or $S \in P Q$ are equally clear.

DEFINITION 2.6. If $\pi_{P, Q, \delta} \cap \pi_{R, T, \delta}=\delta$ or $\pi_{P, Q, \delta}$, we shall say that $\pi_{P, Q, \delta}$ is parallel to $\pi_{R, T, \delta}\left(\pi_{P, Q, \delta} \| \pi_{R, T, \delta}\right)$.

LEMma 2.7. If $\pi_{P, Q, \delta} \| \pi_{R, T, \delta}$ there is an element $\delta^{*}$ of $\delta \ni$ the set of lines of $\pi_{P, Q, \delta}$ incident with $\delta^{*}$ is equal to the set of lines of $\pi_{R, T, \delta}$ incident with $\delta^{*}$.

Proof. Assume $\pi_{P, Q, \delta} \neq \pi_{R, T, \delta}$. Every affine point of $\pi_{R, T, \delta}$ is on a unique line of $\pi_{P, Q, \delta} . \quad \therefore$ Every affine point of $\pi_{R, T, \delta}$ is on a line common to both subplanes. If $l, p$ are common lines, $l \cap p$ is a common point. Thus, $l$ and $p$ are concurrent on $\delta$. Let the point of concurrency be $\delta_{1} \in \delta$.

Let $M$ be an arbitrary point of $\pi_{R, T, \delta} . \quad \exists$ line $l$ of $\pi_{R, T, \delta}$ which is incident with $\hat{o}_{1}$ and $M$. Also $\exists$ line $p \in \pi_{P, Q, \delta}$ which is incident with $M$ and hence $\delta_{1}$. Therefore, the lines $p$ and $l$ are identical.

Thus, the lines of $\pi_{R, T, \delta}$ incident with $\delta_{1} \subseteq$ a set of lines of $\pi_{P, Q, \delta}$ incident with $\delta_{1}$.

The argument is symmetric, so (2.7) is proved.

Lemma 2.8. Let $\pi_{R, T, \delta}$ be any Baer subplane and $P$ a point $\notin$ $\pi_{R, T, \delta}$. Then there is a Baer subplane $\pi_{P, S, \delta}$ containing $\delta$ as a line $\ni$ 
$\pi_{P, S, \dot{o}} \| \pi_{R, T, \dot{0}}$

Proof. Assume without loss of generality that $P R$ is the unique line of $\pi_{R, T, \delta}$ incident with $P$ and assume that $T \notin P R$ (see (2.5)).

Let $S=\left(R T \cap l_{\infty}\right) P \cap\left(P R \cap l_{\infty}\right) T$. Now consider $\pi_{P, S, \delta}$.

Suppose $\pi_{P, S, o} \sharp \pi_{R, T, \delta}$. Let $M$ be a common affine point. Then $M \delta_{i} ; \delta_{i} \in \delta$ are common lines. If $M \notin S T$ then, since $S T$ and $M \delta_{i}$ are common lines, $M \delta_{i} \cap S T$ is a common affine point distinct from $M$ for some $\delta_{i} \in \delta$. But, this is a contradiction by (2.5). $\therefore M \in S T$ and similarly $M \in P R$, which is a contradiction if $M$ is an affine point.

LEMMA 2.9. Let $\pi_{P, Q, \delta}$ be a Baer subplane and suppose $R$ is an affine point $\notin \pi_{P, Q, \delta}$. Then $\exists$ a unique Baer subplane parallel to $\pi_{P, Q, \delta}$ and containing $R$.

Proof. By (2.8) there exists a subplane $\pi_{R, T, \delta} \| \pi_{P, Q, \delta}$.

Suppose $\pi_{0}$ is a Baer subplane with line $\delta$, containing $R$, and $\| \pi_{P, Q, \delta} \cdot$ $\pi_{P, Q, \delta}$ and $\pi_{R, T, \delta}$ have a common concurrent set of lines. Let the point of concurrency be $\delta_{1} \in \delta . \quad R \delta_{1}$ is a line common to $\pi_{0}, \pi_{R, T, \delta}$ and $\pi_{P, Q, \delta}$ so the point of concurrency for the common set of lines of $\pi_{0}$ and $\pi_{P, Q, \delta}$ is also $\delta_{1}$. We can assume without loss of generality that $P Q \cap l_{\infty} \neq \delta_{1}$ since $\pi_{P, \bar{Q}, \bar{o}}=\pi_{P, Q, \bar{o}}$ for any affine point $\bar{Q} \neq P$ of $\pi_{P, Q, \delta}$. By (2.7), $Q \delta_{1}$ is a line of $\pi_{0}, \pi_{R, T, \delta}$ and $\pi_{P, Q, \delta}$ so $\left(P Q \cap l_{\infty}\right) R \cap Q \delta_{1}=D$ is an affine Point $\neq R$ of $\pi_{0}$ and of $\pi_{R, T, \tilde{o}} . \quad \therefore \pi_{0}=\pi_{R, D, \delta}=\pi_{R, T, \delta}$ by (2.5).

Thus, (2.9) is proved.

THEOREM 2.10. (Compare with Ostrom [13], Theorem 5.)

Let $\pi$ be a projective plane. Let $l=l_{\infty}$ be a line of $\pi$ and $\delta$ a derivation set on $l_{\infty}$. Form $\bar{\pi}$ as follows:

$$
\begin{gathered}
\text { points } \bar{\pi}=\text { affine points of } \pi . \\
\text { Lines }\left\{\begin{array}{l}
\text { type } 1=\text { the affine Baer subplanes } \pi_{P, Q, \delta} \\
\text { type } 2=\text { affine restrictions of lines } l \text { of } \pi \ni l \cap l_{\infty} \notin \delta .
\end{array}\right. \\
\| \text {-classes }\left\{\begin{array}{l}
\text { type } 1, \pi_{P, Q, \delta} \| \pi_{R, S, o} \text { iff } \pi_{P, Q, o} \cap \pi_{R, S, o}=\delta \\
\text { type } 2, l \| p \text { iff } l \cap p \in l_{\infty}-\delta .
\end{array}\right.
\end{gathered}
$$

Then $\bar{\pi}$ is an affine plane called the plane derived from $\pi$ by $\delta$.

Proof. Let $P$ and $Q$ be distinct points of $\bar{\pi}$. If $P$ and $Q$ are joined in $\pi$ by $(P Q)_{\pi} \ni(P Q)_{\pi} \cap l_{\infty} \in \delta$ then $\exists 1$ Baer subplane $\pi_{P, Q, \delta}$ containing $P, Q$. If $(P Q)_{\pi} \cap l \notin \delta \exists 1$ line $l$ of $\pi$ containing $P, Q$.

Therefore, two distinct points of $\bar{\pi}$ are uniquely joined.

Let $l$ be a line of $\pi$ such that $l \cap l_{\infty} \notin \delta$ and $\pi_{P, Q, \delta}$ a Baer subplane of $\pi$. Clearly, $l$ must intersect $\pi_{P, Q, \delta}$ in an affine point. 
Thus, for each point $P$ of $\bar{\pi}$ and line $\mathscr{L}$ of $\bar{\pi}$ there is a unique line incident with $P$ and parallel to $\mathscr{L}$.

Thus, $\bar{\pi}$ is an affine plane.

COROLLARY 2.11. Let $l$ be a line of $\pi$ containing distinct affine points $P$ and $Q$ such that $l \cap l_{\infty} \in \delta$. Let $l_{P, Q}=l \cap \pi_{P, Q, \dot{o}}-l \cap l_{\infty}$. Then the points of $l-l \cap l_{\infty}$ and the sets $l_{P, Q}$ as lines form an affine Baer subplane $\bar{\pi}_{P, Q}$ of $\bar{\pi}$.

Proof. Let $R$ and $S$ be any distinct affine points of $l$. $l_{R, S}=$ $l \cap \pi_{R, S, \delta}-l \cap l_{\infty}$ contains $R$ and $S$. Suppose $l_{M, T}$ also contains $R$ and $S$. $l_{M, T}=l \cap \pi_{M, T, \dot{o}}-l \cap l_{\epsilon}$ so that $\pi_{M, T, \tilde{o}}$ contains $R$ and $S$. But $\pi_{M, T, \tilde{o}}=$ $\pi_{R, S, o}$ by (2.5) so that $l_{M, T}=l_{R, S}$. Thus, $R$ and $S$ are uniquely joined.

Let $R$ be any point of $\bar{\pi}_{P, Q}$ not incident with the line $l_{S, T}$. Since $R \in l$ and $l_{S, T}=l \cap \pi_{S, T, \delta}-l \cap l_{\infty}$, then $R \notin \pi_{S, T, \tilde{o}}$. Thus there is a unique Baer subplane $\pi_{R, M, \delta}$ containg $R$ and parallel to $\pi_{S, T, \delta}$. Choose a point $\bar{M}$ of $\pi_{R, M, \delta}$ incident with $l\left(l \cap l_{\infty} \in \delta\right.$ and $R \in l$ so $l$ is a line of $\pi_{R, M, \delta}$ ) and distinct from $R$.

Hence, $\pi_{R, \bar{M}, \dot{v}}=\pi_{R, M, \dot{v}}$ and $l \cap \pi_{R, \bar{M}, \dot{v}}-l \cap l_{\infty}=l_{R, \bar{M}}$ is a line of $\bar{\pi}_{P, Q}$ which is parallel to $l_{S, T}$ and contains $R$. Suppose $l_{N, L}$ is a line of $\bar{\pi}_{P, Q}$ containing $R$ and parallel to $l_{S, T}$.

Now $N L=R \bar{M}=l . \quad \pi_{N, L, \hat{o}}$ and $\pi_{R \bar{M}, \delta}$ have a common line $l$ and a common affine point $R$. Moreover, $\pi_{N, L, \delta}$ and $\pi_{R, \bar{I}, \hat{o}}$ contain no affine points of $l$ in common with $\pi_{S, T, \hat{o}}$.

Suppose $\pi_{N, L, \hat{o}}$ is not parallel to $\pi_{S, T, \tilde{\sigma}}$. Then let $X$ be a common affine point. By assumption, $X \notin l$. Thus, $l$ and $W \delta_{i} \forall \delta_{i} \in \delta$ are lines common to $\pi_{N, L, \hat{o}}$ and $\pi_{S, T, \hat{o}}$. It follows that $S$ and $T$ are points of $\pi_{N, L, o}$ (see (2.4)) so that $\pi_{N, L, \hat{o}}=\pi_{S, T, \hat{o}}$, which is a contradiction.

Thus, both $\pi_{N, L, \bar{o}}$ and $\pi_{R, \bar{H}, \hat{o}}$ are parallel to $\pi_{S, T, \hat{o}}$ and contain $R$ so that $\pi_{N, L, \tilde{o}}=\pi_{R, \bar{\forall}, \delta}$ and hence $l_{N, L}=l_{R, \bar{M}}$. Thus, $\bar{\pi}_{P, Q}$ is an affine subplane of $\bar{\pi}$.

Thus, $l_{P, Q} \| l_{S, T} ; P, Q, S, T$ points of $l$ if and only if $\pi_{P, Q, \delta} \| \pi_{S, T, \tilde{o}}$. Furthermore, given a Baer subplane $\pi_{M, N, \delta}$ not containing a point of $l$, there is a Baer subplane $\pi_{X, Y, \delta}$ with $l$ as a line such that $\pi_{X, Y, \delta} \| \pi_{M, N, \delta}$.

Now extend $\bar{\pi}$ to a projective plane $\bar{\pi}^{*}$. The points on $\bar{l}_{\infty}$ (line at infinity of $\bar{\pi}^{*}$ ) corresponding to the set of all Baer subplanes $\pi_{P, Q, \hat{o}^{*}}$ are precisely the points of $\bar{\pi}_{P, Q}^{*}$.

As a point set $\bar{\pi}_{P, Q}$ is $l-l \cap l_{\infty}$ where $l$ is a line of $\pi$. Therefore, every line of $\bar{\pi}^{*}$ intersects $\bar{\pi}_{P, Q}^{*}$ and every point of $\bar{\pi}^{*}$ is incident with a line of $\bar{\pi}_{P, Q}^{*}$ (that is, a line of $\bar{\pi}_{P, Q}^{*}$ extended to $\bar{\pi}^{*}$. Also note that $l_{P, Q}$ is a subline of $\pi_{P, Q, \delta}$ for $P, Q \in l$ where $\pi_{P, Q, \delta}$ is thought of as a line of $\bar{\pi}$. So the latter statement merely states that every affine point of $\bar{\pi}$ is contained in a Baer subplane $\pi_{P, Q, \delta}$ of $\pi_{\text {.) }}$

Thus, $\bar{\pi}_{P, Q}^{*}$ is a Baer subplane of $\bar{\pi}^{*}$. 
CoRollary 2.12. Let $\pi$ be a projective plane and $\delta$ a derivation set in $l_{\infty}$. Let $\bar{\pi}$ be the affine plane derived from $\pi$ by $\delta$. Then there is a derivation set $\bar{\delta}$ in $\bar{l}_{\infty}$ of the projective extension $\bar{\pi}^{*}$ such that the plane derived from $\bar{\pi}^{*}$ by $\bar{\delta}$ is the affine restriction of $\pi$ by $l_{\infty}$.

Proof. The Baer subplanes $\bar{\pi}_{P, Q}^{*}$ of $\bar{\pi}^{*}$ all have the same set of points $\bar{\delta}$ on $\bar{l}_{\infty}$ (see proof of (2.11)). The affine restrictions of $\bar{\pi}_{P, Q}^{*}$ are affine lines of $\pi$. Clearly, $\bar{\delta}$ is a derivation set in $\bar{l}_{\infty}$.

It is trivial to verify that Baer subplanes are carried into Baer subplanes by collineations.

The following theorem is Ostrom's Theorem 7 and its Corollary [13]. His proofs to these results do not use finiteness in any way.

THEOREM 2.13. (Ostrom [13]). Let $\pi$ be a projective plane and $\delta$ a derivation set on $l_{\infty}$. A collineation $\sigma$ of $\pi \ni \sigma \delta=\delta$ induces $a$ collineation $\bar{\sigma}$ of $\bar{\pi} \ni \bar{\sigma}$ fixes the set $\bar{\delta}$ (the corresponding derivation set of $\left.\bar{l}_{\infty}\right)$. If $\sigma$ is a translation of $\pi, \bar{\sigma}$ is a translation of $\bar{\pi}$.

DEFINITION 2.14. Let $\pi$ be a projective plane and let $l$ be a line of $\pi$. We shall say that $\pi$ is a semi-translation plane with respect to $l$ if and only if $\pi$ admits a group $\mathscr{G}$ of elations with axis $l$, each of whose point orbits along with the set of elation centers for $l$ form a Baer subplane of $\pi$.

$\pi$ is a strict semi-translation (sst) plane with respect to $l$ if $\mathscr{G}$ is the full elation group with axis $l$ and nonstrict (nsst) otherwise.

THEOREM 2.15. (See Ostrom [13].) Let $\pi$ be a projective plane and $l_{\infty}$ a line of $\pi$ and $\delta$ a derivation set in $l_{\infty}$ and let $\bar{\pi}$ denote the affine plane derived from $\pi$ by $\delta$. If $l$ is a line of $\pi$ whose affine restriction is not a line of $\bar{\pi}$ and $\pi$ admits a group of translations $\mathscr{G}$ (elations with axis $l_{\infty}$ ) transitive on the points of $l$, then $\bar{\pi}$ is a semi-translation plane, i.e., $\bar{\pi}^{*}$ (projective extension) is a semi-translation plane with respect to $\bar{l}_{\infty}$.

Proof. By (2.13), since $\mathscr{G} \delta=\delta, \mathscr{G}$ is a group of translations of $\bar{\pi}$. If $l$ is a line of $\pi$ and the restriction of $l$ is not a line of $\bar{\pi}$ then $l-l \cap l_{\infty}$ is an affine Baer subplane of $\bar{\pi}$ (see (2.11)).

Thus we have extended Section III of [13] to arbitrary planes admitting derivations sets. We now consider planes possessing Baer subplanes.

We note that Ostrom's sufficient condition for derivation given in Theorem 9 [13] does not directly apply in the infinite case since 
the indicated affine subplanes are not necessarily Baer subplanes.

3. Baer Subplanes. It is well known and can be easily established by a counting argument that a finite projective plane of order $n$ has Baer subplanes of order $m$ only if $n$ is a square and the order of the subplane is $m=\sqrt{n}$.

For infinite planes no such characterization of Baer subplanes is known. We wish to develop some conditions which are sufficient for a given subplane to be a Baer subplane. For this will use some concepts of André [2] and Bose and Bruck [5].

Definition. Let $V$ be a vector space. A congruence of $V$ is a set $\left\{V_{\alpha}\right\}_{\alpha \in \lambda}$ where $V_{\alpha}$ is a subspace of $V \forall \alpha \in \lambda$ and

$$
\bigcup_{\alpha \in \lambda} V_{\alpha}=V \text { and (2) } V_{\alpha} \oplus V_{\beta}=V \text { for all } \alpha \neq \beta \in \lambda \text {. }
$$

THEOREM 3.2. (André [2]). An affine plane $\pi$ is a translation plane if and only if there is a congruence $\left\{V_{\alpha}\right\}_{\alpha \in \lambda}$ of a vector space $V$ such that the points of $\pi$ are the elements of $V$, the lines of $\pi$ are cosets of elements of $\left\{V_{\alpha}\right\}_{\alpha \in \lambda}$ and the parallel classes are the sets $\left\{V_{\alpha}+b, \alpha\right.$ fixed $\left.\in \lambda, b \in V\right\}$.

THEOREM 3.3. (Lüneburg [11]). Let $\alpha$ be a collineation of a projective plane with axis $l$ and center $P$. Let $Q$ be a point $\neq P$ and $Q \notin l$. Then every projective subplane containing $P, l, Q, Q \alpha$ is left invariant by $\alpha$.

LEMma 3.4. Let $\pi$ be an affine translation plane and $\pi_{0}$ any affine subplane of $\pi$. Then there is a congruence $\left\{V_{\alpha}\right\}_{\alpha \in \lambda}$ for $\pi=V$, a subgroup $W$ of $V$, and subgroups $W_{\alpha}$ of $V_{\alpha}$ for $\alpha \in \lambda^{*} \subseteq \lambda$ such that $\left\{W_{\alpha}\right\}_{\lambda^{*}}$ is a congruence for $W$ which defines $\pi_{0}$.

Proof. Let $P, Q$ be points of $\pi_{0}$. There is a translation $\sigma$ of $\pi$ such that $P \sigma=Q$. By (3.3), $\pi_{0}$ is invariant under $\sigma$.

Clearly, there is a subgroup $\mathscr{T}_{\pi_{0}}$ of the translation group $\mathscr{T}$ of $\pi$ which is sharply transitive on the points of $\pi_{0}$ and leaves $\pi_{0}$ invariant.

Let $\mathscr{T}(P)$ denote the subgroup of $\mathscr{T}$ with fixed center $P \in l_{\infty}$ so that $\mathscr{T}=\bigcup_{P \in l_{\infty}} \mathscr{T}(P) . \quad \mathscr{T}_{\pi_{0}}=\bigcup_{P \in l_{\infty}} \mathscr{T}(P) \cap \mathscr{T}_{\pi_{0}}$. Let $\mathscr{T}(P) \cap$ $\mathscr{T}_{\pi_{0}}=\mathscr{T}_{\pi_{0}}(P)$. Thus, lines of $\pi$ are $\{\mathscr{T}(P)\}_{P \in l_{\infty}}$ and translates of these groups. $\{\mathscr{T}(P)\}_{P \in l_{\infty}}$ and $\left\{\mathscr{T}_{\pi_{\nu}}(P)\right\}_{P \in l_{\infty}}$ are congruences of $\pi$ and $\pi_{0}$, respectively, with the required properties. Note that $W_{\alpha}$ is not necessarily a vector subspace of $V_{\alpha}$ for $\alpha \in \lambda^{*} \cong \lambda$.

Before utilizing (3.4) we mention the following result which depends only on the existence of a particular type of ternary ring. 
Theorem 3.5. Let $Q=(Q,+, \cdot)$ be a ternary ring with ternary function $T$. Let $F=(F,+, \cdot)$ be a sub-ternary ring of $Q$ such that every element of $Q$ can be uniquely written in the form $t \alpha+\beta$ for some $t \in Q-F ; \alpha, \beta \in F$. For all $a, m, b \in Q$ let $T(a, m, b)=t f(a, m$, $b)+g(a, m, b) ; f, g$ functions from $Q \times Q \times Q$ into $F$. Let $f$ and $g$ satisfy properties (1) and (2):

(1) If $m$ and $b$ are fixed and $m \notin F$ there exists an element $a \in F$ such that $f(a, m, b)=0$.

(2) If $a \notin F$ is fixed then $\{(f(a, m, b), g(a, m, b))\}=F \times F$ as $m, b$ vary over $F$.

Then the subplane $\pi^{F}$ coordinatized by $F$ of the plane $\pi^{Q}$ coordinatized by $Q$ is an affine Baer subplane.

Proof. Let $l$ be a line of $\pi^{Q}$. If $l$ is $\{(x, y) \mid x=c$ for $c \in Q\}$ the line $l$ either contains points of $\pi^{F}$ or (in any case) is $\|$ to $\{(x, y) \mid x=$ $\alpha ; \alpha \in F\}$ so the projective extension of $\pi^{Q}$ contains a point of the projective extension of $\pi^{F}$.

If $l$ is $\{(x, y) \mid y=T(x, m, b) ; m, b \in Q\}$ and $m \in F$ then $l \cap l_{\infty}$ is a point of the projective extension of $\pi^{F}$. If $m \notin F$ then by (1) $\exists \alpha \in$ $F \ni f(\alpha, m, b)=0 . \quad \therefore(\alpha, g(\alpha, m, b)) \in\{(x, y) \mid y=T(x, m, b)\} \cap \pi^{F}$.

If $P$ is a point of $\pi^{F}$ let $P=\left(t x_{1}+x_{2}, t y_{1}+y_{2}\right) ; x_{i}, y_{i} \in F$. The lines of $\pi^{F}$ are $\{(x, y) \mid x=\alpha, \alpha \in F\}$ and $\{(x, y) \mid y=T(x, \alpha, \beta) ; \alpha, \beta \in$ $F\}$. If $x_{1} y_{1}=0$ then $P \in\{(x, y) \mid x=\alpha\}$ or $\{(x, y) \mid y=\beta\}$ for some $\alpha$, $\beta \in F$. Thus assume $x_{1} y_{1} \neq 0$. Consider $T\left(t x_{1}+x_{2}, \alpha, \beta\right)$ for some $\alpha$, $\beta \in F$. By (2), $\exists \alpha_{0}, \beta_{0} \ni f\left(t x_{1}+x_{2}, \alpha_{0}, \beta_{0}\right)=y_{1}$ and $g\left(t x_{1}+x_{2}, \alpha_{0}, \beta_{0}\right)=y_{2}$.

CoROLlary 3.6. Let $Q$ be an alternative field and $F$ the associated quaterion skewfield. Then $\pi^{F}$ is a Baer subplane.

Proof. (See Pickert [16], s. 172-3.) $\exists t \in Q \ni a t=t \bar{\alpha} ; \alpha \in F$ and elements of $Q$ are of the form $t \alpha+\beta ; \alpha, \beta \in F$ where $\bar{x}$ denotes a certain involuting automorphism.

$T$ is linear, so $T(\alpha, m, b)=\alpha\left(t m_{1}+m_{2}\right)+t b_{1}+t b_{2}$ (where $m_{i}, b_{i} \in$ $F, i=1,2)=t\left(\bar{\alpha} m_{1}\right)+\alpha m_{2}+t b_{1}+b_{2}=t\left(\bar{\alpha} m_{1}+b_{1}\right)+a m_{1}+b_{2}$. Choose $\bar{\alpha}=-b_{1} m_{1}^{-1}$, then (1) is satisfied. If $\alpha, \beta, \alpha_{i} \in F ; i=1,2$, then $T\left(t \alpha_{1}+\right.$ $\left.a_{2}, \alpha, \beta\right)=\left(t a_{1}+a_{2}\right) \alpha+\beta=\left(t a_{1}\right) \alpha+\left(a_{2} \alpha+\beta\right)=t\left(\alpha a_{1}\right)+a_{2} \alpha+\beta$. (See [16] Pickert, s. 172.) For $\rho, \chi \in F$ and $a_{1} \neq 0 \exists \alpha, \beta \in F \ni \alpha a_{1}=\rho$ and $\chi=a_{2} \alpha+\beta$. $\quad \therefore(2)$ is also satisfied.

We point out that although the Moufang planes contain Baer subplanes it is not clear whether derivation sets exist.

THEOREM 3.7. Let $\pi$ be a translation plane and $\pi_{0}$ a proper subplane. Let $\left\{V_{\alpha}\right\}_{\lambda}$ and $\left\{V_{\alpha}\right\}_{\lambda^{*}}$ be congruences for $\pi$ and $\pi_{0}$, respectively 
where $W_{\alpha}$ is a subgroup of $V_{\alpha}$ and $W$ is a subgroup of $V$ for $\alpha \in \lambda^{*} \varsubsetneqq \lambda$, then if

(i ) (1) $W \cap V_{\delta}=0 \Rightarrow W+V_{\dot{o}}=V$ for each $\delta \in \lambda$, or (2) $V$ and $W$ are finite dimensional over the same skewfield and there is an element $\delta \in \lambda-\lambda^{*}$ such that $W \cap V_{\delta}=0$ and $W+V_{o}=V$, then every line of the projective extension of $\pi$ is incident with a point of the projective extension of $\pi_{0}$.

(ii) Under the assumptions of (i) (1) or (2), $\pi_{0} \nsubseteq \mathbf{U}_{\alpha \in \lambda-\lambda^{*}}\left(V_{\alpha}+b\right)$ for any $b \in V-W$ if and only if $\pi_{0}$ us a Baer subplane.

Proof. First we observe that $V_{\alpha} \cap W=W_{\alpha}$ or 0 depending on whether $\alpha \in \lambda^{*}$ or $\alpha \in \lambda-\lambda^{*}$.

Suppose $V_{\alpha} \cap W \neq 0$ and $\alpha \notin \lambda^{*} . \quad W=\mathbf{U}_{\rho \in \lambda^{*}} W_{\rho} \subseteq \mathbf{U}_{\rho \in \lambda^{*}} V_{\rho}$ and $W_{\rho} \subseteq V_{\rho}$. By assumption, $\exists$ an element $w \in W-\{0\} \ni w \in V_{\alpha}$ and $\alpha \notin$ $\lambda^{*}$. But $w \in V_{\beta}$ for some $\beta \in \lambda^{*} . \quad \therefore V_{\alpha} \cap V_{\beta} \neq 0$, which is a contradiction since $\alpha \neq \beta$.

$\therefore$ If $\alpha \in \lambda-\lambda^{*}, V_{\alpha} \cap W=0$.

Assume $V_{\alpha} \cap W \neq 0$ and $\alpha \in \lambda^{*} . \quad W_{\alpha} \subseteq V_{\alpha} \cap W$ and $W_{\alpha}+W_{\beta}=$ $W ; \alpha, \beta \in \lambda^{*}, \alpha \neq \beta$.

If $c \in W-W_{\alpha}$ then $c=w_{\alpha}+w_{\beta}$ for some $w_{\alpha} \in W_{\alpha}$ and $w_{\beta} \in W_{\beta}-$ $\{0\}$. If $c \in V_{\alpha}$ then $w_{\beta} \in V_{\alpha}$ which is a contradiction. Thus, $W_{\alpha}=$ $V_{\alpha} \cap W$ if $\alpha \in \lambda^{*}$.

For (i) (1), $\delta, \alpha \in \lambda-\lambda^{*} \Rightarrow V_{\delta}+W=V_{\alpha}+W=V$. For (i) (2), $V=$ $V_{o}+W$ is isomorphic to $V_{\alpha}+W \Rightarrow V_{\alpha}+W=V$ for all $\alpha \in \lambda-\lambda^{*}$.

Let $V_{\alpha}+b$ be any line of $\pi$. If $V_{\alpha} \cap W=0$, then $\alpha \in \lambda-\lambda^{*}$ and $V_{\alpha}+W=V$ so $V_{\alpha}+b \cap W \neq \varnothing$. If $V_{\alpha} \cap W \neq 0$, then $V_{\alpha}+b$ for $\alpha \in \lambda^{*}$ is parallel to $V_{\alpha}+w, w \in W$ and since $\left(V_{\alpha} \cap W\right)+w$ is a line of $W=$ $\pi_{0}$, (i) is proved.

If $\pi_{0}=W \nsubseteq \mathbf{U}_{\alpha \in \lambda-\lambda^{*}} V_{\alpha}$ let $b$ be a point of $\pi$. If $b \in \mathbf{U}_{\alpha \in \lambda^{*}} V_{\alpha}$, then $b$ is on a line of $W$. So assume $b \in V-\bigcup_{\alpha \in \lambda^{*}} V_{\alpha}$. Consider the set of lines $V_{\alpha}+b, \alpha \in \lambda$ on $b$. Each $V_{\alpha}+b, \alpha \in \lambda-\lambda^{*}$ intersects $W$ uniquely by the previous argument.

If $W \nsubseteq_{\alpha \in \lambda-\lambda^{*}}\left(V_{a}+b\right) \exists \delta \in \lambda^{*} \ni V_{\delta}+b$ intersects $W$.

$\therefore \pi_{0}$ is an affine Baer subplane. Thus, (ii) is proved.

Let $P G(3, F)$ denote the projective 3 -space over a skewfield $F$. Recall a spread (see Bose and Bruck [4]) $\mathscr{S}$ of $P G(3, F)$ is a covering set of skew lines of $P G(3, F)$.

Barlotti-Bose [3] have studied derivation in dual translation planes of dimension 2 (over their kernels) which correspond to spreads $\mathscr{S}$ of $P G(3, F)$ that have the property that any plane of $P G(3, F)$ contains a line of $\mathscr{S}$ (spreads which are dual spreads). In our terminology this requirement translates to: Let $V_{4}$ be a 4-dimensional vector space over $F$ and $\left\{V_{\alpha}\right\}_{\lambda}$ a congruence for $V_{4}$. Then any 3-dimensional subspace $W$ of $V_{4}$ contains a $V_{\alpha}$ for some $\alpha \in \lambda$. 
REMARK 3.8. Let $V_{4}$ be a 4-dimensional vector space over a skewfield $F$. Let $\left\{V_{\alpha}\right\}_{\lambda}$ be a congruence for $V_{4}$. Then the BarlottiBose assumption is equivalent to asserting that every 2-dimensional subspace of $V_{4}$ which is not a $V_{\alpha}, \alpha \in \lambda$ corresponds to a Baer subplane.

Proof. Let $\Sigma$ be an arbitrary 3-dimensional vector subspace of $V_{4}$ Let $\Sigma_{0}$ be any 2-dimensional subspace of $\Sigma$. Assume $\Sigma_{0}$ is not a $V_{\alpha}, \alpha \in \lambda$. $\Sigma_{0}=\left(\mathrm{U}_{\alpha \in \lambda} V_{\alpha}\right) \cap \Sigma_{0}=\mathrm{U}_{\alpha \in \lambda}\left(V_{\alpha} \cap \Sigma_{0}\right) . \quad V_{\alpha} \cap \Sigma_{0}$ is 1 or 0 dimensional. Define $\lambda^{*}$ as the subset of $\lambda$ such that $V_{\alpha} \cap \Sigma_{0}$ is 1-dimensional. Clearly $\left\{V_{\alpha} \cap \Sigma_{0}\right\}_{\lambda^{*}}$ is a congruence for $\Sigma_{0}$.

Assume the subplane $\pi_{0}$ corresponding to $\left\{V_{\alpha} \cap \Sigma_{0}\right\}_{\lambda^{*}}$ is a Baer subplane. Let $b \in \Sigma-\Sigma_{0}$. Then $b \in V_{\alpha}+r$ for some $\alpha \in \lambda^{*}$ and $r \in \Sigma_{0}$. Since $\Sigma_{0}$ is 2-dimensional, the subspace generated by $b$ and $\Sigma_{0},\left\langle b, \Sigma_{0}\right\rangle=$ $\Sigma$. Since $V_{\alpha} \cap \Sigma_{0}$ is 1-dimensional and $b \notin \Sigma_{0}$ implies that $V_{\alpha} \subseteq\left\langle b, \Sigma_{0}\right\rangle$.

Conversely, assume that every 3 -space of $V_{4}$ contains $V_{\alpha}$ for some $\alpha \in \lambda$. Let $\pi_{0}$ be the subplane corresponding to $\left\{V_{\alpha} \cap \Sigma_{0}\right\}_{\lambda^{*}}$ as above. Since (3.7) (i) (2) holds, we must show that (3.7) (ii) is satisfied. Let $c \in V_{4}-\Sigma_{0}$. By assumption, the subspace $\left\langle-c, \Sigma_{0}\right\rangle$ generated by $-c$ and $\Sigma_{0}$ contains a $V_{\delta}$ for some $\delta \in \lambda$. Clearly, $\delta \in \lambda^{*}$ for otherwise $V_{\delta} \cap \Sigma_{0}=0$. Thus, $c$ is on a line $V_{\delta}+\bar{c}$ of $\pi_{0}$, for $\bar{c} \in \Sigma_{0}$.

We note that Bruen and Fisher [7] have shown that not all spreads of $P G(3, F)$ have the Barlotti-Bose property.

The following theorem also proved by Barlotti and Bose [3] is included. Note that the two arguments are completely distinct.

Definition 3.9. We shall say that a translation plane is of dimension 2 if the corresponding congruence is a 4-dimensional vector space over a skewfield $F$. A dual translation plane shall be said to be of dimension 2 if and only if its dual is of dimension 2 .

THEOREM 3.10. Let $\pi$ be any dual translation plane of dimension 2 such that the corresponding congruence has the property that any 3-space contains a 2-space of the congruence. Then $\pi$ is derivable.

Proof. Let $Q$ be a coordinatizing (left) quasifield for $\pi . \quad Q$ is a right 2-dimensional vector space over $F$ where $F$ is a skewfield contained in the kernel of $Q$. We assert that $\{(\alpha),(\infty), \alpha \in F\} \subseteq l_{\infty}$ of $\pi$ is a derivation set.

It is straightforward to verify that the following sets are subplanes: $\{(a \alpha+b, \alpha \beta+c) ; a \neq 0, b, c$ fixed elements of $Q \forall \alpha, \beta \in F\}$ (see, e.g., Ostrom [13], Theorem 9). By (2.10) it remains to show that they are Baer subplanes.

It is easy to see that the image of a Baer subplane under a 
collineation of the plane is a Baer subplane. We may coordinatize $\pi$ so that $(x, y) \rightarrow(x, y+c)$ for all $c \in Q$ are translations of $\pi$. We need only to consider the subplanes $\{(\alpha \alpha+b, a \beta)\}$.

Let the lines $\{(x, y) \mid y=x m+b\},\{(x, y) \mid x=c\},\{(x, y) \mid y=c\}$ be denoted simply by $y=x m+b, x=c$ and $y=c$, respectively. We may coordinatize the dual plane of $\pi$ by the following: affine points $(m,-b)$ are lines $y=x m+b$ and infinite points $(\infty)$ and $(m), m \in Q$ are lines $l_{\infty}$ and $x=m$, respectively, and conversely. (See, e.g., Fryxell [9].) That is,

$$
\begin{aligned}
(m,-b) & \longleftrightarrow y=x m+b \\
(m) & \longleftrightarrow x=m \\
(\infty) & \longleftrightarrow l_{\infty} .
\end{aligned}
$$

The lines of $\{(\alpha \alpha+b, a \beta)\}$ are $l_{\infty}, y=x \alpha+a \beta-b \alpha$ and $x=a \delta+b$ for $a \neq 0, b$ fixed $\in Q$ and for all $\alpha, \beta \in F$.

The points of the associated dual subplane may be represented by $(\infty),(a \delta+b),(\alpha, b \alpha-a \beta)$ where juxtaposition denotes multiplication in $Q$. Thus if $*$ denotes multiplication in dual $Q$ then the points are $(\infty),(\delta * a+b),(\alpha, \alpha * b-\beta * a)$. Note that $(1, b)$ and $(0, a)$ form a vector basis for the set of affine points so that the affine subplane is a 2dimensional vector subspace and hence is an affine Baer subplane. Since the dual of a Baer subplane is a Baer subplane, (3.10) is proved.

Bruen and Fisher [7] have shown that the condition of (3.9) is valid in any regular or subregular spread of $P G(3, F)$ and, of course, the condition is valid if $F$ is finite. In the finite case, Bruck and Bose [4] have pointed out that subregular spreads correspond to the translation planes constructed by a series of derivations in Desarguesian planes. Note that (3.9) in particular implies that Pappian planes coordinatized by fields $K$ that are quadratic extensions of fields $F$ are derivable. Also, finite André planes of order $q^{2}$ and kern $G F(q)$ may be constructed from Desarguesian planes by a series of derivations. This will be considered in the infinite case.

Lemma 3.11. Let $\pi$ be a Pappian plane. Let $\sigma$ be a nontrivial automorphism of the coordinatizing field $K \ni K$ is a 2-dimensional extension of a field $F$ where the fixed field of $\sigma$ is $F$. Then $\pi_{0}=$ $\left\{(x, y) \mid y=x^{o} m\right\}$ is the set of points of an affine Baer subplane of $\pi$.

Proof. $\pi$ is of dimension 2 and the spread corresponding to $\pi$ is regular (see [4] or [5]). Since $\pi_{0}$ is not a line of $\pi$ and is clearly a 2-dimensional vector space over $F$ it follows from the previous remarks and (3.8) that $\pi_{0}$ is a Baer subplane.

Thus, (3.11) is proved. 
Let $L$ be a field and $\rho$ an automorphism of $L$ with fixed field $L_{\rho}$. If $m \in K$ the norm of $m$ is defined as $\Pi_{\tau \in\langle\rho\rangle} m \tau$. If the order of $\rho$ is finite, an André system with kern $L_{\rho}$ may be defined (see [2] and also [8], p. 355). The lines of the corresponding André plane are cosets (translates) of the sets $\left\{(x, y) \mid y=x^{\rho(m)} m\right\},\{(x, y) \mid x=0\}$ where $\rho(m) \in\langle\rho\rangle$ such that if $m, n \in K$ and $\Pi_{\tau \in\langle\rho\rangle} m \tau=\Pi_{\tau \in\langle\rho\rangle} m \tau$ then $\rho(m)=\rho(n)$.

Lemma 3.12. Let $\pi$ be a Pappian plane coordinatized by a field $K$ which is a 2-dimensional extension of a field $F$. Let $\sigma$ be a nontrivial automorphism of order 2 with fixed field $F$. If $m \in K$ and $\Pi_{\tau \in\langle\rho\rangle} m \tau=m^{1+\sigma}=x \in F$ then $\delta_{x}=\left\{(m) \in \pi \mid m^{1+\sigma}=x\right\}$ is a derivation set in $l_{\infty}$ of $\pi$. The Baer subplanes are the sets $\left\{(x, y) \mid y=x^{\delta} m\right.$ for $\left.m^{1+\sigma}=x\right\}$ and their translates.

Proof. The sets $\left\{(x, y) \mid y=x^{o} m\right\}$ and their cosets are Baer subplanes by (3.11).

Let $P$ and $Q$ be affine points of $\pi$ such that $P Q \cap l_{\infty} \in \delta_{x} . \quad \therefore P Q$ is a line $y=x m+b$ where $m^{1+\sigma}=x ; m, b \in K . \quad P, Q \in y=x m+b$ if and only if $P \tau_{b}, Q \tau_{b} \in y=x m$ where $\tau_{b}$ is the translation represented by $(x, y) \rightarrow(x, y-b)$.

Note that $\left(c^{\sigma-1} m\right)^{1+\sigma}=m^{1+\sigma}=x$. Therefore, $(c, d) \in y=x\left(c^{\sigma-1} m\right)$ if and only if $(c, d) \in y=x^{\sigma} m$.

We can assume without loss of generality that $Q \tau_{b}$ is $(0,0)$. Thus $P \tau_{b},(0,0) \in y=x m$ if and only if $P \tau_{b},(0,0) \in y=x^{\sigma}\left(d^{1-\sigma} m\right)$ for some $d \in K$.

So there is a Baer subplane containing any two points $P$ and $Q$ such that $P Q \cap l_{\infty} \in \delta_{x}$.

Lemma 3.13. (See Bruen and Fisher [7], Theorems 2 and 3.) Let $\mathscr{S}$ be a regular spread in $P G(3, F)$ where $F$ is a field. Let $\mathscr{S}=$ $\bigcup_{i \in \lambda} \mathscr{S}_{i} \cup \mathscr{S}_{0}$ where the $\mathscr{S}_{i} ; i \in \lambda$ are disjoint reguli. Let $\overline{\mathscr{S}}_{i}$ denote the opposite regulus of $\mathscr{S}_{i}$ for all $i \in \lambda$. Then $\overline{\mathscr{S}}=\bigcup_{i \in \lambda} . \overline{\mathscr{S}}_{i} \cup \mathscr{S}_{0}$ is a spread which is a dual spread.

Proof. The argument is essentially the proof of Theorems 2 and 3 of [7]. We shall only sketch the proof.

$\mathscr{S}$ is a dual spread since it is regular. Hence if $\Sigma$ is a plane of $P G(3, F), \Sigma$ contains a line $m$ of $\mathscr{S}$ and hence exactly one. If $m \in \mathscr{S}_{0}$ then $\Sigma$ contains a line of $\overline{\mathscr{S}}$. Therefore, assume $m \in \bigcup_{i \in \lambda} \mathscr{S}_{i}$. Let $m \in \mathscr{S}_{i^{\prime}}$. The lines of $\overline{\mathscr{S}}$ meeting $m$ form a regulus (the opposite regulus to $\left.\mathscr{S}_{i^{\prime}}\right) \overline{\mathscr{S}}_{i^{\prime}}$.

Then, if $p$ and $q$ are lines $\in \mathscr{S}_{i}-\{m\}$ it follows that $(p \cap \Sigma) \cdot(q \cap$ $\Sigma)$ is a line of $\overline{\mathscr{S}}_{i^{\prime}}$. 
By Lemma 12.2 [4], it follows that $\left\{\left\{(x, y) \mid y=x m ; m^{1+\sigma}=x\right\}\right\}$ is a regulus and $\left\{\left\{(x, y) \mid y=x^{\sigma} m ; m^{1+\sigma}=x\right\}\right\}$ its opposite regulus. Thus, each derivation in this case is a matter of "switching" where a regulus is replaced by the opposite regulus. (This is well known in the finite case. See, e.g., [4].)

It appears that there are non-André planes of dim 2 that may be constructed in this way (this is certainly true in the finite casesee Ostrom [12]).

TheOREm 3.14. Any André plane of dimension 2 may be constructed from a Pappian plane by a (possibly infinite) series of derivations.

Corollary 3.15. Any dual André plane of dimension 2 is derivable.

$$
\text { Proof. (3.10), (3.12), (3.13), (3.14). }
$$

THEOREM 3.16. Let $Q$ be any (right) quasifield which is a left 2-dim. vector space over a skewfield $F \subseteq$ Kernel $Q$. Suppose also that $Q$ is a right 2-dim. vector space over $F$. Let $\pi$ be the translation plane coordinatized by $Q$. Let $\pi_{a}=\{(a \alpha, a \beta)$, fixed $a \neq 0 \in Q$ for all $\alpha$, $\beta \in F\} . \quad \pi_{a}$ is a subplane of $\pi$ and $\pi_{a}$ is a right 2-dim. vector subspace of $\pi$ thought of as a (right) 4-dim. vector space over $F$. Suppose there is a skewfield $R \subseteq F$ such that $\forall a \in Q-\{0\} \quad \pi_{a}$ is a left and right vector space of the same finite dimension over $R$. Then $\pi$ is derivable.

Proof. We clearly may extend Ostrom's "homology type" replacement theorem to include the infinite case. (See (3.12), [14].) There is a congruence for $\pi$ which consists of the lines of $\pi$ through the origin. The partial congruence of lines with slopes in $F$ or $(\infty)$ "switches" with the partial congruence of subplanes $\pi_{a}$. It remains to show that we obtain a new congruence and hence a translation plane $\bar{\pi}$ "derived" from $\pi$.

Since $\pi_{a}$ is a left and right vector space of finite dimension $k$ over $R \subseteq F$ and a right vector space of $\operatorname{dim}$. 2 over $F$ then the dimension of $\pi_{a}$ over $R=$ right $\operatorname{dim}\left(\pi_{a} / F\right) \cdot \operatorname{dim} F / R$. Therefore, $\operatorname{dim} F / R=k / 2$.

$\therefore \operatorname{Dim}\{(x, y) \mid y=x m\}$ is $k$ and $\pi_{a}$ and $y=x m, m \notin F$ are independent left $k$-dimensional subspaces over $R$. It follows that we obtain a new congruence over $R$.

Note that it was not required that $\pi_{a}$ be a Baer subplane for the proof. But, since a new congruence is obtained it follows that $\pi_{a}$ is a Baer subplane.

\section{Applications.}

Derivation of Desarguesian Planes. 
By $\S 3$, if $\pi$ is a Pappian plane of $\operatorname{dim} 2$ over a field $K$, then $\pi$ is derivable.

Pickert [17] has given an algebraic construction of the Hall planes which does not require finiteness. Following Albert's [1] theory, the following theorem is clear.

Theorem. (See Pickert [17], Albert [1].) If $\pi$ is a Pappian plane of dimension 2 over a field $K$ then the plane derived from $\pi$ is a Hall plane.

Also note that a spread (congruence) corresponding to $\pi$ must be regular since $\pi$ is Pappian. Clearly then the Barlotti-Bose assumption is valid here. Furthermore, a derivation chain may be constructed on $\pi$ by Barlotti-Bose (see [3] and also [9]).

However, if $\pi$ is a Desarguesian, non-Pappian plane it is not clear that a spread for $\pi$ even contains a regulus. (There are finite spreads which do not contain reguli but, of course, are dual spreads (see, e.g., Bruen [6]).)

\section{The Derivation of the Quaterion Planes.}

The quaterions $Q$ can be considered as a right or left 2-dimensional vector space over the complex $\mathscr{C}$ numbers. Since $\mathscr{C}$ is 2 -dimensional over the reals, (3.16) applies. Thus, the quaterion plane $\pi_{1}$ is derivable.

Consider $\pi_{1} \stackrel{\text { derive }}{\longrightarrow} \pi_{2}$. Clearly $\pi_{2}$ is a translation plane coordinatized by a quasifield $Q_{2}$ (note also that Ostrom's Theorems 9, 10,11 [13] clearly extend to the infinite case in this situation) which is a right and left 2-dimensional vector space over the complex numbers.

That is, let $\{1, t\}$ be a basis for $Q / \mathscr{C}$ so that elements of $Q$ are written in the form $t \alpha+\beta, \alpha, \beta \in \mathscr{C}$. Let $\{1, i, j, k\}$ be the standard basis for $Q$ over the reals.

Let $*$ denote multiplication in $Q_{2}$, then $(\alpha+\beta) * t=t z_{1}+z_{2}$ iff $z_{1} \cdot t=t(\alpha+\beta)+z_{2}$ so $z_{1} t=t \alpha+t \beta+z_{2}$.

Let $z_{1}=a+b i, a, b$ real numbers, and $t=k$ so $(a+b i) k=a k+$ $b(-j)=k a-j b=k(a-i b)$. So $z_{1} t=t \bar{z}_{1}\left(\bar{z}_{1}\right.$ denotes the complex conjugate of $z_{1}$ ).

$\therefore t \bar{z}_{1}=t(\alpha+\beta)+z_{2}$ so $\alpha+\beta=\bar{z}_{1}, z_{2}=0 \overline{\alpha+\beta}=z_{1}$.

$\therefore(\alpha+\beta) * t=t \overline{(\alpha+\beta})=t \alpha+t \beta=\alpha t+\beta t$. So $\alpha * t=\alpha \cdot t$. It follows also that $\alpha * a=\alpha \cdot \alpha$ for all $\alpha \in \mathscr{C}$ and $a \in Q$.

Thus, $Q_{2}$ is $2-\operatorname{dim} / \mathscr{C}, \mathscr{C}$ is the kernel of $Q_{2}$ and $Q_{2}$ is also right 2-dim over $\mathscr{C}$.

It is fairly easy to verify that multiplication $*$ in $\pi_{2}$ may be obtained as:

$$
(t \alpha+\beta) *(t \delta+\gamma)=t \overline{\left(\beta-\alpha \delta^{-1} \gamma\right)} \delta+\overline{\left(\beta-\alpha \delta^{-1} \gamma\right)} \gamma-\overline{\alpha \delta^{-1}} .
$$


From this equation the mult $*$ can be defined in terms of the basis $\{1, i, j, k\}$.

Some open questions here are: $\pi_{1}$ ?

(1) Is the full collineation group of $\pi_{2}$ the group inherited from

(2) Is $\pi_{2}$ a previously known plane?

Let $\pi_{a}=\{(\alpha \alpha, \alpha \beta)\}, a=t a_{1}+a_{2} ; a_{i} \in \mathscr{C}$ and $a_{1} a_{2} \neq 0$. Then if $\rho \in$ $\mathscr{C}, \rho a=a \delta$ for some $\delta \in \mathscr{C}$ if and only if $\bar{\rho}=\rho$. Thus, $\pi_{a}$ is a right and left vector subspace of dimension 4 over the reals but is not, in general, a left subspace over the complex numbers.

\section{The Derivation of André Planes}

I. Nearfield planes. Sabharwal [20] has constructed a class of infinite nearfield planes (which are André planes). Each nearfield is of dimension 2 over its kernel where the kernel is a finite extension by radicals of the rationals.

By theorem (3.15) the dual planes are derivable. Actually, Sabharwal shows that a derivation chain can be based on these planes. Moreover, he shows how to construct infinite analogues of the Hughes planes and considers a derivation chain on such planes.

Sabharwal's description is essentially given as follows: Let $F=$ $Q(\sqrt{p})$ where $Q$ is the field of rationals and $p$ is a positive nonsquare in $Q$.

Define multiplication

$$
x \circ y=\left\{\begin{array}{l}
x y \text { if the norm } x=x^{1+\sigma} \geqq 0 \\
x y^{\sigma} \text { if } x^{1+\sigma}<0
\end{array}\right.
$$

where $\sigma$ is the automorphism $\sqrt{p} \stackrel{\sigma}{\longrightarrow}-\sqrt{p}$.

II. Bol planes. Burn [8] has given an example of an infinite Bol quasifield $Q$ which is an André system. Both the plane $\pi$ coordinatized by $Q$ and its dual are derivable by (3.15) and (3.16). Moreover, it appears that a derivation chain may be constructed on $\pi$ (see [8], pp. 356-357).

Semifield planes. Infinite weak nucleus semifields may be constructed analogous to the Hughes-Kleinfeld-Knuth finite semifields (see [10]) which be derivable by (3.16).

Because of space, we shall postpone explication of the derived planes of this section to a later paper. The discussion of "nets" has been avoided in this treatment, although the set of Baer subplanes of a derivable plane form lines of a net. In the finite case the union of two disjoint nets on the same points form a net. However, in the 


\section{infinite situation this has yet to be proved.}

\section{REFERENCES}

1. A. A. Albert, The finite planes of Ostrom, Bol. Soc. Mat. Mexicana, (1967), 1-13.

2. J. André, Über nicht-Desarguessche Ebenen mit transitiver Translations gruppe, Math. Z., 60 (1954), 156-186.

3. A. Barlotti and R. C. Bose, Linear Representation of a Class of Projective Planes in Four Dimensional Projective Space, Inst. Statistics Mimeo Ser. No. 600.20, Dept. Stat., Univ. North Carolina at Chapel Hill (1970).

4. R. C. Bose and R. H. Bruck, The construction of translation planes from projective spaces, J. Algebra, I (1964), 85-102.

5 . - Linear representations of projective planes in projective spaces, J. Algebra, 4 (1966), 117-172.

6. A. Bruen, Spreads and a conjecture of Bruck and Bose, To appear in J. Algebra.

7. A. Bruen and J. C. Fisher, Spreads which are not dual spreads, Canad. Math. Bull, 12 (1969), No. 6, 801-803.

8. R. P. Burn, Bol quasi-field and Pappus' theorem, Math. Z., 105 (1968), 351-364.

9. R. Fryxell, Sequences of planes constructed from nearfield planes of square order, Ph. D. dissertation, Washington State Univ., 1964.

10. D. E. Knuth, Finite semifields and projective planes, J. Algebra, 2 (1965), 182-217.

11. H. Lüneburg, Charakterisierungen der endlichen desarguesschen projektiven Ebenen, Math. S., 85 (1964), 419-450.

12. T. G. Ostrom, Finite Tpanslation Planes, Springer-Verlag Notes $\# 158$ (1970).

13. - Semi-translation planes, Trans. Amer. Math. Soc., 111 (1964), 1-18.

14. - Vector spaces and construction of finite projective planes, Arch. Math., 19 (1968), 1-25.

15. G. Panella, Una classe di sistemi cartesiani, Atti. Accad. Naz. Lincei Rend. Cl. Fis. Mat. Natur., 8 (1965), 480-485.

16. G. Pickert, Projektive Ebenen, Springer-Verlag, Berlin (1955).

17. - Die cartesischen Gruppen der Ostrom-Rosati-Ebenen, Abh. Hamburg, 30 (1967), 106-117.

18. L. A. Rosati, Su una generalizzuzione dei piani di Hughes., Atti Accad. Naz. Lincei Rend. Cl. Fis. Mat. Natur., 29 (1960), 330-308.

19. - Su una nuova classe di piani grafici, Ricerche Mat., 13 (1964), 39-55.

20. R. Sabharwal, Planes associated with infinite nearfields, Ph. D. Dissertation, Washington State Univ., 1966.

21. J. D. Swift, Chains and graphs of Ostrom planes, Pacific J. Math., 14 (1964), 353362.

Received July 7, 1971 and in revised form July 13, 1972. This work was supported (in part) by grant no. GP-27445, National Science Foundation.

The UNIVERsity of IOWA 


\section{PACIFIC JOURNAL OF MATHEMATICS}

\section{EDITORS}

\author{
H. SAMELSON \\ Stanford University \\ Stanford, California 94305 \\ C. R. HOBBY \\ University of Washington \\ Seattle, Washington 98105
}

\author{
J. DugundJI \\ Department of Mathematics \\ University of Southern California \\ Los Angeles, California 90007
}

\author{
RICHARD ARENS \\ University of California \\ Los Angeles, California 90024
}

\section{ASSOCIATE EDITORS}
E. F. BECKENBACH
B. H. NeumanN
F. WOLF
K. YoSHIDA

\section{SUPPORTING INSTITUTIONS}

\author{
UNIVERSITY OF BRITISH COLUMBIA \\ CALIFORNIA INSTITUTE OF TECHNOLOGY \\ UNIVERSITY OF CALIFORNIA \\ MONTANA STATE UNIVERSITY \\ UNIVERSITY OF NEVADA \\ NEW MEXICO STATE UNIVERSITY \\ OREGON STATE UNIVERSITY \\ UNIVERSITY OF OREGON \\ OSAKA UNIVERSITY
}

\author{
UNIVERSITY OF SOUTHERN CALIFORNIA \\ STANFORD UNIVERSITY \\ UNIVERSITY OF TOKYO \\ UNIVERSITY OF UTAH \\ WASHINGTON STATE UNIVERSITY \\ UNIVERSITY OF WASHINGTON \\ $* * * *$
$*$
AMERICAN MATHEMATICAL SOCIETY
NAVAL WEAPONS CENTER
}

The Supporting Institutions listed above contribute to the cost of publication of this Journal, but they are not owners or publishers and have no responsibility for its content or policies.

Mathematical papers intended for publication in the Pacific Journal of Mathematics should be in typed form or offset-reproduced, (not dittoed), double spaced with large margins. Underline Greek letters in red, German in green, and script in blue. The first paragraph or two must be capable of being used separately as a synopsis of the entire paper. The editorial "we" must not be used in the synopsis, and items of the bibliography should not be cited there unless absolutely necessary, in which case they must be identified by author and Journal, rather than by item number. Manuscripts, in duplicate if possible, may be sent to any one of the four editors. Please classify according to the scheme of Math. Rev. Index to Vol. 39. All other communications to the editors should be addressed to the managing editor, Richard Arens, University of California, Los Angeles, California, 90024.

50 reprints are provided free for each article; additional copies may be obtained at cost in multiples of 50 .

The Pacific Journal of Mathematics is published monthly. Effective with Volume 16 the price per volume (3 numbers) is $\$ 8.00$; single issues, $\$ 3.00$. Special price for current issues to individual faculty members of supporting institutions and to individual members of the American Mathematical Society: $\$ 4.00$ per volume; single issues $\$ 1.50$. Back numbers are available.

Subscriptions, orders for back numbers, and changes of address should be sent to Pacific Journal of Mathematics, 103 Highland Boulevard, Berkeley, California, 94708.

PUBLISHED BY PACIFIC JOURNAL OF MATHEMATICS, A NON-PROFIT CORPORATION

Printed at Kokusai Bunken Insatsusha (International Academic Printing Co., Ltd.), 270, 3-chome Totsuka-cho, Shinjuku-ku, Tokyo 160, Japan. 


\section{Pacific Journal of Mathematics}

\section{Vol. 43, No. 2 \\ April, 1972}

Arne P. Baartz and Gary Glenn Miller, Souslin's conjecture as a problem on the real line....................................... 277

Joseph Barback, On solutions in the regressive isols ............... 283

Barry H. Dayton, Homotopy and algebraic K-theory ................ 297

William Richard Derrick, Weighted convergence in length ............ 307

M. V. Deshpande and N. E. Joshi, Collectively compact and semi-compact sets of linear operators in topological vector spaces ............. 317

Samuel Ebenstein, Some $H^{p}$ spaces which are uncomplemented in $L^{p} \ldots . .327$

David Fremlin, On the completion of locally solid vector lattices ......... 341

Herbert Paul Halpern, Essential central spectrum and range for elements of

a von Neumann algebra............................... 349

G. D. Johnson, Superadditivity intervals and Boas' test ............. 381

Norman Lloyd Johnson, Derivation in infinite planes . . . . . . . . . . . 387

V. M. Klassen, The disappearing closed set property .............. 403

B. Kuttner and B. N. Sahney, On the absolute matrix summability of Fourier series ........................................... 407

George Maxwell, Algebras of normal matrices................... 421

Kelly Denis McKennon, Multipliers of type $(p, p) \ldots \ldots \ldots \ldots \ldots \ldots . . \ldots 29$

James Miller, Sequences of quasi-subordinate functions ............. 437

Leonhard Miller, The Hasse-Witt-matrix of special projective varieties ..... 443

Michael Cannon Mooney, A theorem on bounded analytic functions ...... 457

M. Ann Piech, Differential equations on abstract Wiener space .......... 465

Robert Piziak, Sesquilinear forms in infinite dimensions ............. 475

Muril Lynn Robertson, The equation $y^{\prime}(t)=F(t, y(g(t))) \ldots \ldots \ldots \ldots .483$

Leland Edward Rogers, Continua in which only semi-aposyndetic

subcontinua separate ............................... 493

Linda Preiss Rothschild, Bi-invariant pseudo-local operators on Lie

groups ...................................... 503

Raymond Earl Smithson and L. E. Ward, The fixed point property for

arcwise connected spaces: a correction ...................... 511

Linda Ruth Sons, Zeros of sums of series with Hadamard gaps .......... 515

Arne Stray, Interpolation sets for uniform algebras............... 525

Alessandro Figà-Talamanca and John Frederick Price, Applications of random Fourier series over compact groups to Fourier multipliers .. 